\title{
Postnatal Involution and Counter-Involution of the Thymus
}

\author{
Jennifer E. Cowan ${ }^{1 *}$, Yousuke Takahama ${ }^{2}$, Avinash Bhandoola ${ }^{1}$ and Izumi Ohigashi ${ }^{3 *}$ \\ ${ }^{1}$ Laboratory of Genome Integrity, Center for Cancer Research, National Cancer Institute, National Institutes of Health, \\ Bethesda, MD, United States, ${ }^{2}$ Experimental Immunology Branch, National Cancer Institute, National Institutes of Health, \\ Bethesda, MD, United States, ${ }^{3}$ Division of Experimental Immunology, Institute of Advanced Medical Sciences, University of \\ Tokushima, Tokushima, Japan
}

Thymus involution occurs in all vertebrates. It is thought to impact on immune responses in the aged, and in other clinical circumstances such as bone marrow transplantation. Determinants of thymus growth and size are beginning to be identified. Ectopic expression of factors like cyclin D1 and Myc in thymic epithelial cells (TEC)s results in considerable increase in thymus size. These models provide useful experimental tools that allow thymus function to be understood. In future, understanding TEC-specific controllers of growth will provide new approaches to thymus regeneration.

\section{OPEN ACCESS}

Edited by:

Ann Chidgey,

Monash University, Australia

Reviewed by:

Adrian Liston

Flanders Institute for Biotechnology, Belgium

Daniel Gray,

Walter and Eliza Hall Institute of

Medical Research, Australia

${ }^{*}$ Correspondence:

Jennifer E. Cowan

jennifer.cowan@nih.gov

Izumi Ohigashi

ohigashi@genome.tokushima-u.ac.jp

Specialty section: This article was submitted to

T Cell Biology,

a section of the journal

Frontiers in Immunology

Received: 20 February 2020 Accepted: 17 April 2020 Published: 12 May 2020

Citation:

Cowan JE, Takahama Y, Bhandoola A and Ohigashi I (2020) Postnatal

Involution and Counter-Involution of the Thymus. Front. Immunol. 11:897.

doi: 10.3389/fimmu.2020.00897
Keywords: thymus, Myc, cyclin D1, growth, involution, aging

\section{INTRODUCTION}

The thymus is the primary site of T cell development. It is a specialized environment that fosters the production of a diverse $\mathrm{T}$ cell repertoire, allowing $\mathrm{T}$ cells to recognize and eliminate foreign antigen but remain tolerant to self. The thymic epithelial cells (TEC)s educate developing $\mathrm{T}$ cells, where cortical (c)TECs select for those expressing functional receptors, whilst medullary (m)TECs deplete those with potential specificity to the body's own cells (1). TECs provide essential signals to $\mathrm{T}$ cell precursors that drive their migration, differentiation, proliferation, and survival. Correspondingly, developing thymocytes provide signals to drive TEC differentiation and organization. This process is termed thymic crosstalk $(1,2)$. Impaired TEC development results in severe $\mathrm{T}$ cell immunodeficiency (3-5).

\section{THYMIC INVOLUTION}

The thymus is the first organ to undergo aged-related involution and at an accelerated rate relative to other tissue (6). The process of thymic involution is evolutionarily conserved in all vertebrates (7). The organ undergoes rapid growth during development, peaks in size around adolescence and begins to decline with age; with the initiation of involution beginning as early as birth and no later than the onset of puberty in humans and mice (6). This thymic regression includes reductions in thymic mass, loss of thymic structure, and disorganization to thymic architecture, consequently resulting in diminished thymocyte numbers and reduced naïve T cell output $(6,8,9)$. In addition to chronic age-related involution, the thymus can undergo acute atrophy under conditions of physiological stress, such as infection (10), pregnancy $(11,12)$, and cancer treatments (13).

Stressed-induced thymic involution results in decreased naïve $\mathrm{T}$ cell output and compromised host immunity, and is commonly reversible, with recovery of size and function after the insult is removed $(6,14,15)$. This acute involution involves rapid reductions in proliferation and enhanced apoptosis of developing thymocytes. These impairments in developing $\mathrm{T}$ cells can be a direct effect of infection on thymocytes (16). However, acute thymic involution can also be a consequence of the 
infectious agent on TEC, which in turn drives thymocyte death $(16,17)$. An example of the latter is seen with synthetic dsRNA Poly(I:C) treatment, which mimics a viral infection and rapidly triggers thymic involution in mice. This acute thymic atrophy is mediated by type I IFN responses, that do not act directly on the T cell precursors (17).

Signals between TEC and thymocytes are bi-directional, with defects and alterations in the haemopoietic component of the thymus also having dramatic consequences on TEC development and maintenance. The importance of lymphoid-epithelial cell interactions for thymic architecture was established in mice lacking mature T cells, that present with severely impaired mTEC development (18). Early stage blocks in T cell development, such as in human $\mathrm{CD} 3 \varepsilon$ transgenic mice, show severely disrupted thymic architecture and defective cTEC and mTEC development (19), whilst mice with later stage defects in the TCR complex, such as genetic disruptions to the TCR $\alpha$ gene, Rag-1 deficiency or ZAP-70, have severely abrogated mTEC generation (1921). Interesting, successful reconstitution in the adult thymus does not require thymic crosstalk in the fetal period. Thus, adult human $\mathrm{CD} 3 \varepsilon$ transgenic mice receiving WT fetal liver transplants had increased thymic size and restored thymic architecture and function (22). As thymocyte-derived signals are so essential in the establishment and maintenance of the thymic microenvironment, a role for both lymphoid cells and epithelial cells needs to be considered in the process of stressed-induced and chronic age-related involution.

Many agents have been identified to be involved in acute stress-induced thymic atrophy, including proinflammatory cytokines, steroids, and hormones. Inhibition of such agents can prevent this acute involution (14). The drivers of chronic age-related involution remain less clear, and it is unknown if the same cellular and molecular mechanisms underlie both chronic and acute involution (6). Sex hormones, increased after puberty, facilitate the chronic involution process, and castration of old mice can successfully albeit transiently restore both thymic size and function (23). Moreover, castration of young adult mice resulted in enhanced thymic regeneration following bone marrow transplantation (24). Sex steroid ablation can also enhance thymic function in humans. Prostate cancer patients undergoing sex steroid ablation therapy present with significant increases in numbers of naive $\mathrm{T}$ cells as a consequence of enhanced thymic function and T cell export (24). Although it is still poorly understood how sex steroids drive age-related thymic involution, atrophy is at least partially attributed to increased sex steroids at puberty (25).

\section{IMMUNOLOGICAL CONSEQUENCES OF THYMIC INVOLUTION}

Although aging results in widespread immunodeficiency, the direct consequences of age-related thymic involution on impaired immune function in the elderly is poorly understood. Old people are more susceptible to infection, and infections in the elderly often have higher severity compared to the young (8). Furthermore, the elderly mount poorer responses to vaccines (26) and show increased incidence of cancers (27). These phenomena are suggested to be, at least in part, a consequence of declining numbers and diversity of naive T cells emerging from the aged thymus, which in turn contributes to the shift toward memory phenotypes in the periphery $(8,26,27)$.

Age-related thymic involution in mouse limits the numbers of recent thymic emigrants in the periphery (28). Moreover, in humans, using T-cell receptor excision circles (TREC) to measure thymic output, generation of new $\mathrm{T}$ cells was minimal in the elderly, with the frequency of TREC declining steadily with age $(29,30)$, although the precise age of when this reduction is initiated remains unclear (31). This was confirmed in a study that acquired thymic samples in addition to peripheral blood (32). Elderly individuals displayed a wide range of thymic functionality, measured by the frequency of double positive thymocytes (the higher the frequency of DP, the higher the functionality). When thymic function was compared to percentages of naïve $\mathrm{T}$ cells, reduced thymus function correlated well with decreased naïve $\mathrm{T}$ cell numbers. Moreover, this comparison identified a strong relationship between the low functioning thymi and the contraction of naïve CD8 T cells specifically. These studies also revealed a relationship between thymic function and the dynamics of peripheral naive $\mathrm{T}$ cells, where reduced thymus function was associated with shortened telomere length and aberrant activation and proliferation status in naïve CD8 T cells (32).

There are examples of viruses that aged mice are more susceptible to, as a consequence of age-related alteration to $\mathrm{T}$ cell responses. These include West Nile Virus, where old mice have reduced rates of survival compared to young. This increased susceptibility is a consequence of age-related defects in $\mathrm{T}$ cell immunity, involving both CD8 and CD4 T cell responses (33). Influenza has also been identified as an age-sensitive virus. It has been demonstrated that compromised protective immunity in aging against influenza can be a consequence of the ageassociated decline in CD8 $\mathrm{T}$ cell repertoire diversity, resulting in "holes" in the repertoire that may impair the ability of T cells to control the virus (34).

Rejuvenation of the involuted thymus restores peripheral $\mathrm{T}$ cell function in aged mice and humans (24, 35). Many agents have been identified to reverse thymic atrophy, although their effects are only transient. Those include interleukin-7, sex steroids, growth hormones, and keratinocyte growth factor (KGF) (36). Sex steroid ablation can also improve immune responses. The level of restoration, however, following castration is limited by age, with enhancement of thymic function following the procedure lost between 9 and 18 months. Nine-monthold castrated males when challenged with Influenza A display restored numbers of virus specific cytotoxic CD8 T cells to levels comparable to young mice, and improved viral clearance in the lung (37). In contrast, 24-month-old castrated mice did not display restored numbers of virus-specific CD8 T cells to young levels, yet still had observed reductions in lung viral titers (37). Thus, age-associated defects in $\mathrm{T}$ cell immunity may, at least in part, be attributed to the diminished naïve $\mathrm{T}$ cell repertoires, and thymic regeneration by sex steroid ablation can restore this deficiency and improve viral immune 
responses, but in an age-limited manner. Moreover, sex steroid ablation must be having additional T-cell independent effects that improve viral clearance (37). Interestingly, a recent publication has suggested that restoration of thymic size in an aging host using sex steroid ablation or KGF does not confer protection to the host against West Niles Virus (38). Thus, modest increases to thymic size in aging hosts might not be sufficient to improve immune protection. The complete contribution of thymic involution to age-associated defects in $\mathrm{T}$ cell immunity remains to be determined.

Overall the immunological consequences of age-related thymic involution on the peripheral $\mathrm{T}$ cell pool potentially leaves the elderly with compromised protective immunity against pathogens. Thus, a better understanding of the molecular and cellular mechanisms underlining the causes of age-related thymic involution should offer benefits to the aging population in multiple clinical settings. It also needs to be considered, however, that age associated immune system defects may also be due to the aging of the hematopoietic cells, and not all a consequence of reduced thymic function. The general physiological consequences of aging to the host also needs to be considered when exploring the attributes of compromised protection against infectious agents in the elderly.

\section{THE ROLE OF TECs IN THYMIC INVOLUTION}

It has been reported that the transfer of bone marrow cells or early $\mathrm{T}$ cell progenitors isolated from young mice does not restore thymic function in aged recipients, whereas the transplantation of fetal thymi into aged hosts exhibits thymocyte development equivalent to a young host. Thus, indicating that thymic stromal cells, rather than hematopoietic cells, drive thymic involution (15, 39-41). The reduction in size of the aged thymus is accompanied with reduced TEC numbers (9). Postnatal reductions in expression of the Forkhead box N1 (Foxn1) transcription factor is sufficient to accelerate age related thymic involution (42, 43). Moreover, targeted overexpression of Foxn 1 in adult TECs can attenuate the involution process and delay the decline in naive $\mathrm{T}$ cells observed in the aging host (35). Foxn1 expression is diminished in aged stroma (44), suggesting a relationship between the decline of Foxn 1 expression and age-associated thymic involution.

It has been demonstrated that genetic ablation of cTECs alone has profound effects on thymic size, and severely impaired thymocyte development (45). This complements a recent publication that explored the morphological changes in TECs with age and suggested dramatic changes to cTEC structure alone reduce thymic size (46). The authors genetically labeled TECs using conditional confetti mice to facilitate the visualization of individual TECs in cortical or medullary regions. First it was established that cTECs in the thymus of young mice have unique morphology, with extensive networks of projections estimated to engulf over 100 lymphocytes within them. Second, these cTEC projections contracted in the thymus of 12-month mice, in contrast to mTEC morphology, that is unaltered with age. As the authors suggest, this dramatic alteration to cTEC morphology may contribute to thymic involution, instead of or in addition to increased cTEC death or decreased rates of CTEC proliferation with age (46). This morphological characterization of adult cTECs further offers a possible explanation as to why conventional enzymatic digestion methods used for cTEC isolation dramatically underestimate their cellularity in the postnatal thymus (47).

In addition to morphological changes with age in cTECs, their expression of Catalase, an antioxidative enzyme, is lower compared to mTECs and lymphocytes, which results in their increased susceptibility to oxidative damage (48). Indeed, the morphological alteration of cTECs is greatest in the sub-capsular cortex, which is the region of intense proliferation activity of lymphocytes, and thus of intense metabolism (46).

Transcriptional profiling of TECs in young and aged thymi has revealed dynamic gene profiles during the initial involution process in both cTECs and mTECs, aiding the understanding of the mechanisms that govern the decline of TEC numbers with age. A transcriptional hallmark of the initiation of involution in TECs was the downregulation of genes involved in cell cycle, specifically diminished E2F3 activity, suggesting possible reductions to cell cycle progression in all aging TECs (49). The same aging-associated transcriptional changes are seen in mTECs during the initial phase of thymic involution, from 2 to 10 weeks of age (50). Furthermore, gene expression profiles of cTECs display greater alterations during involution and regeneration, than those of both their mTEC counterparts and developing $\mathrm{T}$ cells (23). The dynamic changes in CTEC transcriptomes following castration mediated thymic regeneration revealed that genes upregulated in expression during thymic regrowth included those involved in cell morphology, cell adhesion, and cytoskeleton remodeling (46).

The underlying mechanisms of the age-related decline in TEC numbers and function that mediate thymic atrophy remain ambiguous, although many changes to TEC biology throughout life have been documented. The pivotal role of TECs in the process of thymic atrophy identify them as an attractive therapeutic target to counter thymic involution (15). Although the morphological, metabolic and transcriptional alterations with age are more prevalent in CTECs, the significance of this has yet to be determined. One major constraint of conclusively identifying a role for cTECs in age-associated thymic involution is the limitation in the numbers of cTECs able to be isolated from the adult thymus. We hope the enlarged thymus models discussed in this review will provide useful experimental tools to isolate greater numbers of adult cTECs to perform more detailed analysis of the changes that occur in this population with age. In addition, the newly refined genetic models described below will facilitate the ectopic expression of Myc specifically in cTEC or mTEC subsets (51). This could potentially determine whether such targeted manipulation is sufficient to reverse involution and force thymus growth. 


\section{TECs MEDIATE CHANGES TO THYMIC SIZE THROUGHOUT LIFE}

In addition to controlling thymic atrophy, TECs regulate all changes in thymic size observed throughout life. This includes the dramatic increase seen during mouse embryogenesis, when the thymus doubles in size daily, and the continued expansion, although at a reduced rate, until 4 weeks of age, when thymic cellularity peaks. TECs also maintain this maximal size until around 8 weeks of age, when involution is initiated (9). Defects in TEC development and numbers results in diminished thymic size (52). Moreover, dramatic expansion in TEC numbers in adulthood results in simultaneous expansion in the numbers of developing lymphocytes and severe thymic hyperplasia (51, 53-55). Correspondingly, and as mentioned previously, postnatal disruption of TECs triggers accelerated thymic atrophy $(42,43)$. Furthermore, restricting the Foxn 1 dependent TEC progenitors results in reduced thymic size during the initial formation of the thymus, and this persists into the adult period (56).

The rapid expansion in thymic size and TEC number during fetal and neonatal life is supported by TECs having unique properties that distinguish them from postnatal populations. This includes higher rates of proliferation $(9,51)$ and ribosomal biogenesis (51). Additionally, fetal TECs are functionally distinct compared to their adult counterparts. This includes their ability to undergo successful engraftment following intrathymic injection into an adult host, a property lost after birth (57). In addition, fetal TECs have an exclusive ability to support development of fetal waves of gamma delta $\mathrm{T}$ cells (58). Moreover, it has also been suggested that TEC progenitors in the fetal and postnatal thymus differ. TECs in the adult thymus have a turnover rate of $\sim 2$ weeks (9), yet their putative self-renewal capacity and precursor- product relationships remain unclear $(1,2)$. A common bipotent TEC progenitor has been identified at E13.5 that can give rise to both cTECs and mTECs $(59,60)$. During initial thymus formation, TEC progenitors can first acquire some cTEC specific markers, before differentiating into the mTEC lineage $(2,61)$, but mTEC lineage restricted progenitors have also been identified in the fetal thymic environment (62). Corresponding progenitor populations in the adult thymus remain to be determined (2).

\section{Myc ACTIVITY IN TECs IS LIMITING FOR THYMIC SIZE AND FUNCTION}

Understanding the transcriptional controllers of fetal TECs and how they support the rapid expansion in thymic size during embryogenesis offers insight into mechanisms underlying thymic function and regeneration. Recently, transcriptional assessment of TECs through development at a single cell resolution has revealed distinct transcriptional programs of TECs at specific stages of life $(51,63-65)$. This included the identification of a fetal specific program comprising high levels of expression of Myc target genes, including cell cycle genes and genes involved in ribosomal biogenesis. Such genes displayed declining expression in TECs through fetal development, coordinated with reduced expression of Myc protein. Transcript levels corresponded with declining rates of cell proliferation and correlates of ribosomal biogenesis in TECs through development. It was hypothesized that this high Myc activity in fetal TECs drives the expansion in TEC numbers and consequently thymic size during early development (Figure 1A) (51). It has previously been reported that mice with conditional removal of Myc in TECs present with small thymi in adulthood, as a consequence of reduced rates of proliferation and decreased TEC numbers (66). Consistently, enhancing Myc expression in TECs resulted in a severe increase in TEC number and dramatic thymic hyperplasia in adulthood (Figure 1B). This continued Myc expression in TECs conferred expression of a fetal-specific transcriptional program, including high levels of expression of genes involved in cell cycle and ribosomal biogenesis (51). Interestingly, this ribosomal signature was unique to the Myc model, as another transgenic large thymus model, driven by cell cycle gene cyclin D1, did not present with increased transcripts of genes involved ribosomal biogenesis $(51,67)$.

The dramatic thymic growth conferred by the Myc transgene did not jeopardize function, similar to previous reports with cyclin D1 transgenic mice (53, 54), and such enlarged thymi also produced increased numbers of recent thymic emigrants. Furthermore, this continued Myc expression preserved the ability of TECs to engraft following intrathymic injection into adulthood. Myc overexpression did not need to be maintained throughout life to increase thymic size, with inducible expression of Myc in adult and aged TECs being sufficient to similarly promote thymic growth (Figure 1C). Although the decline in Myc activity was most striking between fetal and adult TECs, the same reductions, but at much more modest rates, could be detected transcriptionally between adult and aged cTEC populations (51). Thus, this decline in Myc activity in TECs could underlie the reduction in thymic cellularity observed during involution.

Collectively, these results identify Myc as a major regulator of a fetal specific transcriptional program of TECs. They establish a role for Myc activity in rapid thymic growth during development; provide evidence Myc expression can confer at least one functional distinct property of fetal TECs into adulthood and furthermore, confirm that Myc activity is limiting for thymic size and function (51). Upstream controllers of Myc in TECs remain to be identified and are logical candidates in modulating thymic function and regeneration.

In addition to unique transcriptional profiles of fetal TECs, the adult TECs were highly enriched for genes involved in antigen processing and presentation, along with regulation of the actin cytoskeleton and the lysosomes (51). Our results suggest an adult specific transcriptional program of TECs supports the maintenance of a functional thymus in adulthood, complementing and extending previous work $(49,63,64)$. 

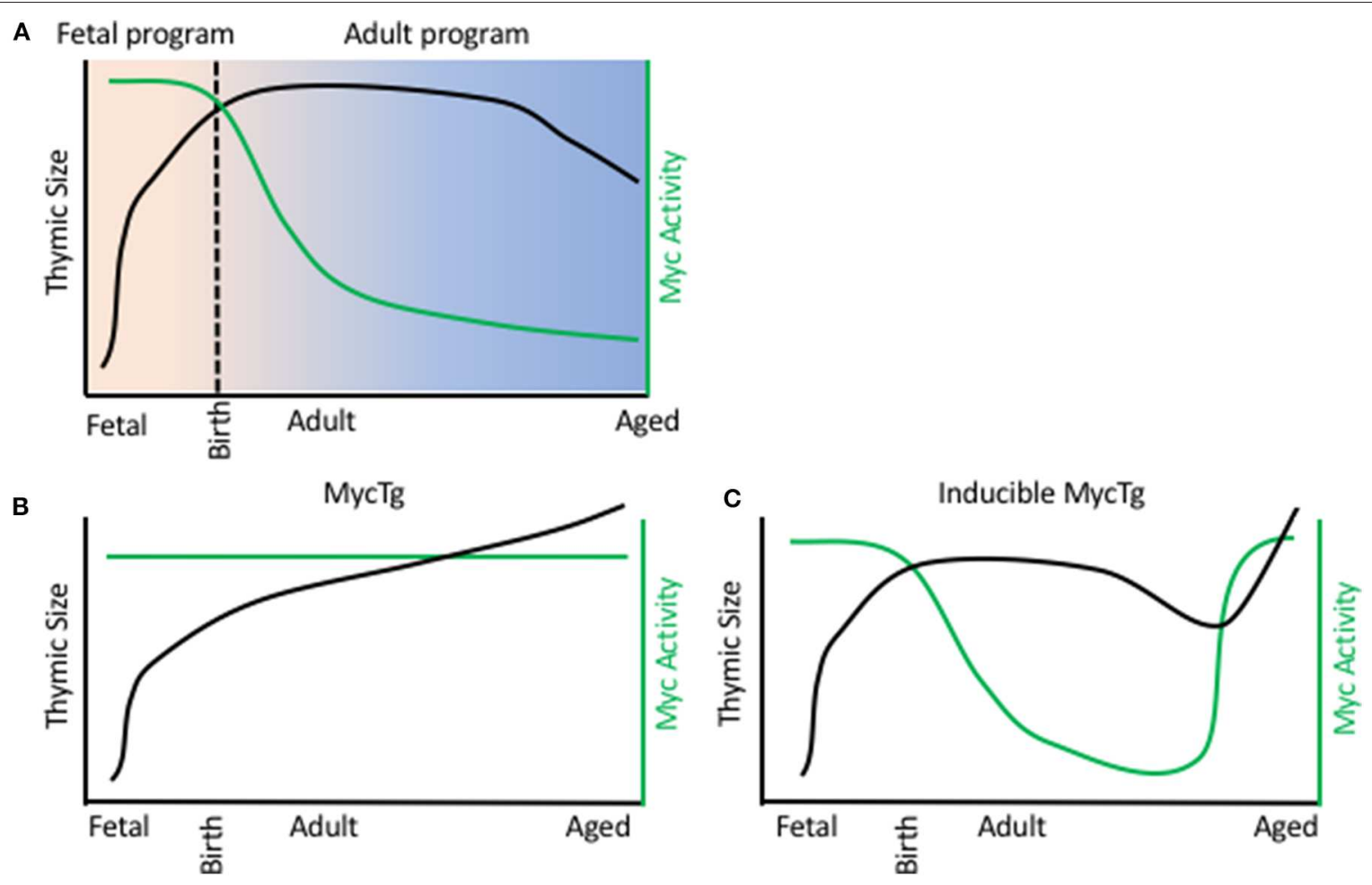

FIGURE 1 | Myc activity in TEC limits thymic size. (A) Thymic size (black line) and Myc activity in TECs (green line) during a WT mouse life span. X axis represents age, from day of fertilization. B/C. Thymic size (black line) and Myc activity in TECs (green line) during a FoxN1 MycTg mouse (B) or an inducible MycTg mouse (C) life span.

\section{CYCLIN D1 OVEREXPRESSION IN THYMIC EPITHELIAL CELLS ENLARGES FUNCTIONAL THYMUS}

Cyclin family molecules are key regulators for cell cycle progression that control the number of cells and the size of organs. Cyclin proteins associate and activate cyclin-dependent kinases (CDKs), serine/threonine kinases, important for the progression of cell cycle (68). Approximately 30 members of cyclin family proteins, defined by the cyclin box domain, are known in human and mice $(69,70)$. Among the cyclin family proteins, cyclin A, cyclin B, cyclin D, and cyclin E are canonical cyclins, which interact with cell-cycle-related CDKs and regulate cell division $(71,72)$. These canonical cyclins regulate distinct phases of cell cycle; cyclin A regulates S and G2 phases, cyclin $B$ regulates $M$ phase, cyclin $D$ regulates $G 1$ phase, and cyclin $E$ regulates $\mathrm{G} 1$ and $\mathrm{S}$ phases (73-76).

Cyclin D1 is a member of cyclin D family, along with cyclin D2 and cyclin D3. Overexpression of cyclin D1 in cells results in a rapid progression from $\mathrm{G} 1$ to $\mathrm{S}$ phase, whereas inhibition of cyclin D1 prevents the entry into S phase (77). The overexpression of cyclin D1 in mouse increases the incidence of carcinoma in the mammary gland and the liver $(78,79)$. Correlation between elevated expression of cyclin D1 and various cancers and their poor prognosis is also noted in human $(80,81)$ and mouse (82).
To investigate the role of cyclin D1 in epithelial cells, Robles et al. generated transgenic mice in that cyclin D1, encoded by human CCND1 gene, was driven by bovine keratin 5 promoter (53). Keratin 5 is primarily expressed in basal keratinocytes of the skin epidermis. It was found that these keratin 5-driven cyclin D1-transgenic (K5D1) mice exhibit epidermal hyperplasia in the skin (53). The authors also found that the thymus in K5D1 mice exhibit severe hyperplasia, which is apparent by 14 weeks of age when age-associated thymic involution is detectable in control mice, whereas the thymus in K5D1 mice at 2 weeks of age is comparable in size to that in control mice (53). In the thymus, TEC progenitors and mTECs highly express keratin $5(54,83)$, and both the cortical and medullary regions are enlarged in the thymus of K5D1 mice $(53,54)$.

The enlarged thymuses in K5D1 mice contain $\sim 100$-fold larger numbers of TECs compared with control C57BL/6 (B6) thymuses, and the cellularity of thymocytes in K5D1 mice reaches to $\sim 50$-fold larger numbers of that in B6 mice $(67,84)$. In K5D1 mice, the cellularity of cTECs is elevated similarly to that of mTECs, although keratin 5 is predominantly detectable in mTECs but not cTECs (67). It is possible that the increase in the number of cTECs is at least in part due to cyclin D1-mediated promotion of cell cycle in keratin 5-expressing TEC progenitors (54). It is additionally possible that cell cycle progression within cTEC 


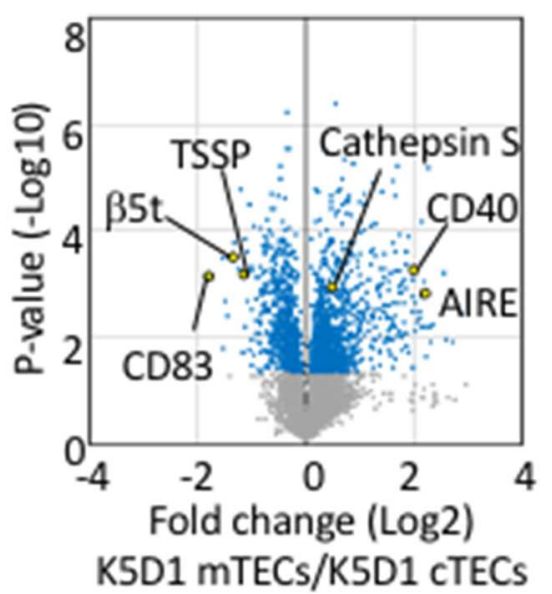

\section{5,753 proteins quantified}

1,021 proteins significantly higher in $\mathrm{MTECS}$ 636 proteins significantly higher in CTECS

FIGURE 2 | Proteomic analysis of TECs isolated from enlarged thymus. Volcano plot of TMT-based quantitative proteomes for CTECs and mTECs isolated from keratin 5 promoter-driven cyclin D1-transgenic (K5D1) mice. Detected proteins, which we have recently reported (67), are plotted as log2 fold changes (K5D1 cTECs / K5D1 mTECs) vs. -log10 P-values.

compartment is promoted in K5D1 mice, since a remarkably elevated amount of cyclin D1 transcripts are detected in K5D1 cTECs (67). The signals provided by developing thymocytes play an important role in the development of cTECs (19, 83). Indeed, hCD3 $\varepsilon$ transgenic mice, in which early thymocyte development is primarily defective, do not exhibit thymic hyperplasia even when they are crossed with K5D1 mice to overexpress cyclin D1 in TECs (54). Thus, developing thymocytes appear to contribute to the increase in TEC cellularity in K5D1 mice.

K5D1 thymuses support thymocyte development similar to $\mathrm{B} 6$ thymuses, as the proportion of developing thymocytes subsets, including $\mathrm{CD} 4^{-} \mathrm{CD} 8^{-}, \mathrm{CD} 4^{+} \mathrm{CD}^{+}, \mathrm{CD} 4^{+} \mathrm{CD} 8^{-}$, and $\mathrm{CD} 4^{-} \mathrm{CD} 8^{+}$thymocytes in K5D1 mice is almost comparable to that in $\mathrm{B} 6$ mice (67). However, mature CD4- or CD8-single positive thymocytes identified as $\mathrm{CD}^{-} 9^{-} \mathrm{Qa}^{+}$are accumulated in the K5D1 thymus (84). This accumulation is possibly due to unelevated amount of sphingosine-1-phosphate (S1P), produced by unelevated number of endothelial cells in the K5D1 thymus, because S1P plays an essential role in promoting thymic egress of mature thymocytes (85). Indeed, in contrast to thymocytes, which are $\sim 50$-fold-elevated in cell number, the increase of splenic T cell number in K5D1 mice is only 2 - to 3 -fold of that in B6 mice $(67,84)$. In addition to the possible limitation in the machinery for thymic egress of mature thymocytes, limited availability of cytokines, such as Interleukin-7 (IL-7), to maintain peripheral $\mathrm{T}$ cells, may also limit the cellularity of the peripheral $\mathrm{T}$ cell pool in K5D1 mice (86).

\section{THYMIC EPITHELIAL CELLS IN CYCLIN D1-MEDIATED ENLARGED THYMUS ARE FUNCTIONALLY POTENT}

The function of the thymus to produce self-protective $\mathrm{T}$ cells and to instill their self-tolerance is chiefly mediated by cTECs and mTECs, respectively. The capability of thymocyte development in K5D1 mice suggests the functional equivalence between K5D1 TECs and B6 TECs. cTECs uniquely express $\beta 5 \mathrm{t}-$ containing thymoproteasome important for optimal production of immunocompetent $\mathrm{CD}^{+} \mathrm{T}$ cells (87-89), whereas Aire expressed in mTECs plays a role for the establishment of selftolerance in $\mathrm{T}$ cells (90). The enlarged K5D1 thymuses contain $\beta 5 \mathrm{t}^{+}$cTECs in the cortex and Aire ${ }^{+}$mTECs in the medulla (67). It was shown that $\beta 5$ t deficiency in K5D1 mice results in the impaired generation of $\mathrm{CD} 8^{+} \mathrm{T}$ cells in the thymus, and the loss of Aire in mTECs causes autoimmune inflammation in various tissues, including the retinas and salivary glands, in K5D1 mice (67). $\mathrm{T}$ cells generated in the K5D1 thymus show a proliferative response to allogenic stimulation and are unresponsive to synergic cells (67). T cells that express TCR specific for a male specific $\mathrm{H}-\mathrm{Y}$ antigen are positively selected in the thymus of female K5D1 mice, whereas those $\mathrm{T}$ cells are negatively selected in the thymus of male K5D1 mice (54). Thus, cTECs and mTECs in the enlarged K5D1 thymus are functionally competent to produce immunocompetent yet selftolerant $\mathrm{T}$ cells.

\section{PROTEOMIC PROFILING OF THYMIC EPITHELIAL CELLS ISOLATED FROM CYCLIN D1 ENLARGED THYMUS}

Isolation of cTECs and mTECs generally involves enzymatic digestion of thymic tissues. Although the thymus in one postnatal B6 mouse contains more than $1 \times 10^{6}$ cTECs and more than $1 \times 10^{6}$ mTECs, the number of either cTECs or mTECs isolated from one B6 mouse is typically $<1 \times 10^{4}$ cells $(46,47)$. However, a large number of cells (typically more than $5 \times 10^{5}$ cells per sample) are required for current mass spectrometry-based technology of proteomic analysis, unlike transcriptomic analysis, which can be carried out from small number of cells. Using the enlarged thymus in K5D1 mice, it is possible to isolate $\sim 2 \times$ $10^{5}$ cTECs and $\sim 2 \times 10^{5}$ mTECs from one mouse (67). These relatively large numbers of cTECs and mTECs allow unbiased proteomic analysis.

cTECs and mTECs isolated from K5D1 mice are qualified for proteomic analysis, because RNA-sequencing-based transcriptomic profiles of cTECs and mTECs are highly similar between K5D1 mice and B6 mice (67). For example, functionally relevant genes in mTECs, including Aire, Ccl21a, and Tnfrsfl1a (encoding RANK), as well as Aire-dependent and Aire-independent tissue-restricted self-antigen genes are highly detected in mTECs in an indistinguishable manner between K5D1 mTECs and B6 mTECs (67). Similarly, functionally relevant genes in cTECs, including Dll4 (encoding DLL4), Psmb11 (encoding $\beta 5$ t), Prss16 (encoding thymus-specific serine 
protease or TSSP), and Ctsl (encoding cathepsin L), are highly detected in cTECs in an indistinguishable manner between K5D1 cTECs and B6 cTECs (67). Only a minor difference in transcriptomic profiles between K5D1 TECs and B6 TECs is the overexpression of cyclin D1 and cell-cycle-related genes (67). Another minor difference detected between K5D1 TECs and B6 TECs is the difference in the number of $\mathrm{CD} 4^{+} \mathrm{CD} 8^{+}$ thymocytes tightly associated with isolated cTECs (67). cTECs form multicellular complexes that a single cTEC completely envelops a variety number of $\mathrm{CD} 4^{+} \mathrm{CD}^{+}$thymocytes $(46,91)$. These multicellular complexes include thymic nurse cells, which entirely encapsulate $\mathrm{CD} 4^{+} \mathrm{CD} 8{ }^{+}$thymocytes (91) and so cannot be fully dissociated from encapsulated $\mathrm{CD} 4^{+} \mathrm{CD} 8^{+}$thymocytes by current technology for cTEC isolation (67). The number of enclosed DP thymocytes per one isolated cTEC is smaller in K5D1 mice than $\mathrm{B} 6$ mice, so that transcriptomic profiles of K5D1 cTECs is less affected than those in B6 cTECs by the signals derived from $\mathrm{CD} 4{ }^{+} \mathrm{CD} 8^{+}$thymocytes (67). Nevertheless, cTECs and mTECs isolated from K5D1 mice are not only functionally potent to produce immunocompetent and self-tolerant $\mathrm{T}$ cells but also are highly similar in transcriptomic profiles, so that cTECs and mTECs isolated from K5D1 mice are largely qualified for proteomic profiling to better understand the biology of cTECs and mTECs.

Tandem mass tag (TMT)-based mass spectrometry analysis is a powerful technology for unbiased proteomics that enables multiplex analysis of relative quantification of proteins (92). It successfully quantified 5,753 protein species in total in cTECs and mTECs isolated from K5D1 mice by the TMT-based quantitative proteomic analysis (67). Similar to the transcriptomic profiles, proteomic profiles show a sharp contrast between cTECs and mTECs; 636 proteins, including $\beta 5 t$, TSSP, and CD83, which are known to play an important role in cTECs, are significantly ( $p$ $<0.05$ ) higher in cTECs than mTECs, whereas 1,021 proteins, including Aire, cathepsin S, and CD40, which are known to play an important role in mTECs, are significantly $(p<0.05)$ higher in mTECs than cTECs (Figure 2). It was noticed that secretory proteins, including cytokines and chemokines, are removed from isolated cTECs and mTECs during cell isolation procedures and are undetectable in the proteomic profiles (67).

These proteomic profiles for cTECs and mTECs have led to integrated analysis of proteomic and transcriptomic profiles in cTECs or mTECs (67). Approximately $70 \%$ of molecules detected in proteomic analysis of K5D1 TECs are detected in transcriptomic profiles of B6 TECs, whereas 30\% of molecules detected in transcriptomic profiles of B6 TECs are detected in proteomic analysis of K5D1 TECs (67). These overlapped molecules include many molecules previously reported in TECs and include molecules such as DCLK1, Avil, and Trmp5, expressed by thymic tuft cells, a recently described subpopulation of mTECs $(64,65,67)$. Newly identified molecules include cathepsin D and calpain 1, abundantly expressed in cTECs, and cathepsin $\mathrm{C}$, cathepsin $\mathrm{H}$, and cathepsin $\mathrm{Z}$, abundant in mTECs (67). These proteases may play an important role in processing self-antigens in cTECs and mTECs and in enabling TCR repertoire selection in the thymus, in addition to previously described $\beta 5 \mathrm{t}$, TSSP, and cathepsin L in cTECs, and cathepsin $\mathrm{S}$ in mTECs. The integrated analyses of K5D1 TECs further reveal that genetic loss of $\beta 5 \mathrm{t}$ specifically alters the amount of individual proteasomal components in cTECs but minimally affects proteomic and transcriptomic profiles in cTECs (67).

It is interesting to note that proteomic profiles and transcriptomic profiles do not provide a proportional correlation between the amount of proteins and mRNAs in cTECs and mTECs (67). It is possible that post-transcriptional turnover of transcripts and post-translational turnover of proteins may account for the disagreement between the abundance of mRNAs and proteins (67). Thus, quantitative proteomic profiling of TECs isolated from $\mathrm{K} 5 \mathrm{D} 1$ mice has revealed a previously unknown platform for further exploring molecular biology of TECs.

Propagation of mobilized cell lines that maintain functionally relevant molecular expression profiles has not been successful for cTECs or mTECs, so that the enlarged thymus provides a useful source of large numbers of freshly isolated cTECs and mTECs, not only for proteomic analysis but also for other analyses, including metabolomic analysis and other biochemical analyses. It is certainly important to identify MHC-associated self-peptides presented by cTECs and mTECs, which induce positive and negative selection of thymocytes to form an immunocompetent yet self-tolerant TCR repertoire.

\section{WHY DOES THE THYMUS INVOLUTE?}

Although the immunological consequences of thymic involution have potential disadvantages to the elderly, thymic involution may have evolved for the benefit of the aging host. However, such benefits may be less favorable given current lifespans. One hypothesis is that reduced thymic activity would protect against autoimmunity (93). Seemingly at odds with this idea, accelerated thymic involution in young mice can disrupt central immune tolerance, by perturbed negative selection resulting in the release of autoreactive $\mathrm{T}$ cells into the periphery $(94,95)$. However, experimental systems could conceivably differ from naturally occurring thymic involution. Separately, it is known that some $\mathrm{T}$ cells in elderly mice that express markers of self-recognition are selectively retained during aging $(96,97)$. This could offer an explanation as to why the elderly have a predisposition to certain autoimmune conditions $(94,97)$, that could be mitigated by thymic involution. Overall, considerably more work is needed to determine whether the elderly are indeed at increased risk for autoimmunity, and whether reduced thymic activity may have any protective function.

It has also been suggested the involution process may conserve energy. The majority of developing thymocytes die during the stringent selection process in the thymus, where over $90 \%$ are estimated to undergo cell death (98), and the longlived peripheral $\mathrm{T}$ cells established in early life can undergo homeostatic proliferation to maintain their numbers (99). Thus, energy may be best diverged into other biological processes after the T cell pool is established in early life (6).

Another possibility is that the reduced numbers of naïve $\mathrm{T}$ cells may also reduce the incidence of leukemias (6). Others have 
suggested the diminished numbers of recent thymic emigrants from the involuting thymus forces enhanced selection of the peripheral T cell repertoire and conserves the persistence of longlived memory $\mathrm{T}$ cells in the periphery which are favorable to the host in old age $(99,100)$. If such theories are correct and involution is beneficial to the host, approaches to restore thymic size and function in old people become questionable. Therefore, a better understanding of whether thymic involution is favorable to the host will establish if thymic regeneration would be beneficial as a clinical approach.

\section{CONCLUSIONS AND PERSPECTIVES}

T cell-mediated immunity is essential throughout life. However, the thymus, where $\mathrm{T}$ cells are generated, involutes rapidly in early life. Postnatal thymic involution is attributable to TECs rather than thymocytes. In this review, we summarize recent findings that the decline in a fetal-specific transcriptional program of TECs controls the size of the postnatal thymus and that cTEC morphology is altered during postnatal thymic involution. These findings provide novel insights into molecular and cellular mechanisms in TECs that control thymus size during involution. Thymic involution results in reduced thymocyte development and reduced numbers of naïve $\mathrm{T}$ cells and so is predicted to result in immunological disadvantages, including increased incidence in infectious disease and delayed $\mathrm{T}$ cell reconstitution after hematopoietic stem cell transplantation (101). Therefore, prevention of thymic involution and regeneration of thymic

\section{REFERENCES}

1. Kadouri N, Nevo S, Goldfarb Y, Abramson J. Thymic epithelial cell heterogeneity: TEC by TEC. Nat Rev Immunol. (2019) 20:239-53. doi: 10.1038/s41577-019-0238-0

2. Takahama Y, Ohigashi I, Baik S, Anderson G. Generation of diversity in thymic epithelial cells. Nat Rev Immunol. (2017) 17:295-305. doi: $10.1038 /$ nri.2017.12

3. Nehls M, Pfeifer D, Schorpp M, Hedrich H, Boehm T. New member of the winged-helix protein family disrupted in mouse and rat nude mutations. Nature. (1994) 372:103-7. doi: 10.1038/372103a0

4. Adriani M, Martinez-Mir A, Fusco F, Busiello R, Frank J, Telese $\mathrm{S}$, et al. Ancestral founder mutation of the nude (FOXN1) gene in congenital severe combined immunodeficiency associated with alopecia in southern Italy population. Ann Hum Genet. (2004) 68:265-8. doi: 10.1046/j.1529-8817.2004.00091.x

5. Larsen BM, Cowan JE, Wang Y, Tanaka Y, Zhao Y, Voisin B, et al. Identification of an intronic regulatory element necessary for tissue-specific expression of foxn1 in thymic epithelial cells. J Immunol. (2019) 203:686-95. doi: 10.4049/jimmunol.1801540

6. Shanley DP, Aw D, Manley NR, Palmer DB. An evolutionary perspective on the mechanisms of immunosenescence. Trends Immunol. (2009) 30:374-81. doi: 10.1016/j.it.2009.05.001

7. Torroba M, Zapata AG. Aging of the vertebrate immune system. Microsc Res Tech. (2003) 62:477-81. doi: 10.1002/jemt.10409

8. Gui J, Mustachio LM, Su DM, Craig RW. Thymus size and age-related thymic involution: early programming, sexual dimorphism, progenitors and stroma. Aging Dis. (2012) 3:280-90.

9. Gray DH, Seach $\mathrm{N}$, Ueno $\mathrm{T}$, Milton MK, Liston A, Lew $\mathrm{AM}$, et al. Developmental kinetics, turnover, and stimulatory function is likely to be useful for the maintenance and improvement of T-cell-mediated immune homeostasis. On the other hand, the increase in the number of peripheral $\mathrm{T}$ cells is limited in mice that carry an enlarged thymus, suggesting that merely enhancing the number of TECs and the size of the thymus is insufficient to expand the peripheral $\mathrm{T}$ cell pool.

In this review, we also summarize how the development of large thymus models are useful tools for understanding TEC biology. This includes the versatility of enlarged K5D1 thymi for biochemical analysis, including proteomic profiling of TECs. Using such useful tools, we can examine whether functional senescence is induced in enlarged thymi and how counterinvolution in aged mice may impact health. Moreover, such models could give novel targets for manipulation to prevent or reverse thymus atrophy.

\section{AUTHOR CONTRIBUTIONS}

The review was written by JC and IO and was revised by YT and $\mathrm{AB}$.

\section{FUNDING}

This research was supported by the Intramural Research Program of the Center for Cancer Research at the National Cancer Institute, and by the NCI-NIA Joint Fellowship on Cancer and Aging. capacity of thymic epithelial cells. Blood. (2006) 108:3777-85. doi: 10.1182/blood-2006-02-004531

10. Savino W. The thymus is a common target organ in infectious diseases. PLoS Pathog. (2006) 2:e62. doi: 10.1371/journal.ppat.0020062

11. Clarke AG, Kendall MD. The thymus in pregnancy: the interplay of neural, endocrine and immune influences. Immunol Today. (1994) 15:545-51. doi: 10.1016/0167-5699(94)90212-7

12. Laan M, Haljasorg U, Kisand K, Salumets A, Peterson P. Pregnancy-induced thymic involution is associated with suppression of chemokines essential for T-lymphoid progenitor homing. Eur J Immunol. (2016) 46:2008-17. doi: 10.1002/eji.201646309

13. Fletcher AL, Lowen TE, Sakkal S, Reiseger JJ, Hammett MV, Seach N, et al. Ablation and regeneration of tolerance-inducing medullary thymic epithelial cells after cyclosporine, cyclophosphamide, and dexamethasone treatment. $J$ Immunol. (2009) 183:823-31. doi: 10.4049/jimmunol.0900225

14. Gruver AL, Sempowski GD. Cytokines, leptin, and stress-induced thymic atrophy. J Leukoc Biol. (2008) 84:915-23. doi: 10.1189/jlb.0108025

15. Chaudhry MS, Velardi E, Dudakov JA, van den Brink MR. Thymus: the next (re)generation. Immunol Rev. (2016) 271:56-71. doi: 10.1111/imr. 12418

16. Dooley J, Liston A. Molecular control over thymic involution: from cytokines and microRNA to aging and adipose tissue. Eur J Immunol. (2012) 42:1073-9 doi: 10.1002/eji.201142305

17. Anz D, Thaler R, Stephan N, Waibler Z, Trauscheid MJ, Scholz $\mathrm{C}$, et al. Activation of melanoma differentiation-associated gene 5 causes rapid involution of the thymus. J Immunol. (2009) 182:6044-50. doi: 10.4049/jimmunol.0803809

18. Shores EW, Van Ewijk W, Singer A. Disorganization and restoration of thymic medullary epithelial cells in $\mathrm{T}$ cell receptor-negative scid mice: evidence that receptor-bearing lymphocytes influence maturation 
of the thymic microenvironment. Eur J Immunol. (1991) 21:1657-61. doi: 10.1002/eji.1830210711

19. Hollander GA, Wang B, Nichogiannopoulou A, Platenburg PP, van Ewijk W, Burakoff SJ, et al. Developmental control point in induction of thymic cortex regulated by a subpopulation of prothymocytes. Nature. (1995) 373:350-3. doi: $10.1038 / 373350 \mathrm{a} 0$

20. Palmer DB, Viney JL, Ritter MA, Hayday AC, Owen MJ. Expression of the alpha beta T-cell receptor is necessary for the generation of the thymic medulla. Dev Immunol. (1993) 3:175-9. doi: 10.1155/1993/56290

21. Negishi I, Motoyama N, Nakayama K, Nakayama K, Senju S, Hatakeyama S, et al. Essential role for ZAP-70 in both positive and negative selection of thymocytes. Nature. (1995) 376:435-8. doi: 10.1038/376435a0

22. Roberts NA, Desanti GE, Withers DR, Scott HR, Jenkinson WE, Lane $\mathrm{PJ}$, et al. Absence of thymus crosstalk in the fetus does not preclude hematopoietic induction of a functional thymus in the adult. Eur J Immunol. (2009) 39:2395-402. doi: 10.1002/eji.200939501

23. Griffith AV, Fallahi M, Venables T, Petrie HT. Persistent degenerative changes in thymic organ function revealed by an inducible model of organ regrowth. Aging Cell. (2012) 11:169-77. doi: 10.1111/j.1474-9726.2011.00773.x

24. Sutherland JS, Goldberg GL, Hammett MV, Uldrich AP, Berzins SP, Heng TS, et al. Activation of thymic regeneration in mice and humans following androgen blockade. J Immunol. (2005) 175:2741-53. doi: 10.4049/jimmunol.175.4.2741

25. Velardi E, Dudakov JA, van den Brink MR. Sex steroid ablation: an immunoregenerative strategy for immunocompromised patients. Bone Marrow Transplant. (2015) 50(Suppl. 2):S77-81. doi: 10.1038/bmt.2015.101

26. Wagner A, Garner-Spitzer E, Jasinska J, Kollaritsch H, Stiasny K, Kundi M, et al. Age-related differences in humoral and cellular immune responses after primary immunisation: indications for stratified vaccination schedules. $S c i$ Rep. (2018) 8:9825. doi: 10.1038/s41598-018-28111-8

27. Palmer S, Albergante L, Blackburn CC, Newman TJ. Thymic involution and rising disease incidence with age. Proc Natl Acad Sci USA. (2018) 115:1883-8. doi: 10.1073/pnas.1714478115

28. Hale JS, Boursalian TE, Turk GL, Fink PJ. Thymic output in aged mice. Proc Natl Acad Sci USA. (2006) 103:8447-52. doi: 10.1073/pnas.0601040103

29. Douek DC, McFarland RD, Keiser PH, Gage EA, Massey JM, Haynes BF, et al. Changes in thymic function with age and during the treatment of HIV infection. Nature. (1998) 396:690-5. doi: 10.1038/25374

30. Naylor K, Li G, Vallejo AN, Lee WW, Koetz K, Bryl E, et al. The influence of age on T cell generation and TCR diversity. J Immunol. (2005) 174:7446-52. doi: 10.4049/jimmunol.174.11.7446

31. Mitchell WA, Lang PO, Aspinall R. Tracing thymic output in older individuals. Clin Exp Imuunol. (2010) 131:497-503. doi: $10.1111 / j .1365-2249.2010 .04209 . x$

32. Ferrando-Martinez S, Ruiz-Mateos E, Hernandez A, Gutierrez E, Rodriguez-Mendez Mdel M, Ordonez A, et al. Age-related deregulation of naive T cell homeostasis in elderly humans. Age. (2011) 33:197-207. doi: 10.1007/s11357-010-9170-8

33. Brien JD, Uhrlaub JL, Hirsch A, Wiley CA, Nikolich-Zugich J. Key role of T cell defects in age-related vulnerability to west nile virus. J Exp Med. (2009) 206:2735-45. doi: 10.1084/jem.20090222

34. Yager EJ, Ahmed M, Lanzer K, Randall TD, Woodland DL, Blackman MA. Age-associated decline in $\mathrm{T}$ cell repertoire diversity leads to holes in the repertoire and impaired immunity to influenza virus. J Exp Med. (2008) 205:711-23. doi: 10.1084/jem.20071140

35. Zook EC, Krishack PA, Zhang S, Zeleznik-Le NJ, Firulli AB, Witte PL, et al. Overexpression of Foxn1 attenuates age-associated thymic involution and prevents the expansion of peripheral CD4 memory T cells. Blood. (2011) 118:5723-31. doi: 10.1182/blood-2011-03-342097

36. Dorshkind K, Montecino-Rodriguez E, Signer RA. The ageing immune system: is it ever too old to become young again? Nat Rev Immunol. (2009) 9:57-62. doi: 10.1038/nri2471

37. Heng TS, Reiseger JJ, Fletcher AL, Leggatt GR, White OJ, Vlahos K, et al. Impact of sex steroid ablation on viral, tumour and vaccine responses in aged mice. PLoS ONE. (2012) 7:e42677. doi: 10.1371/journal.pone.0042677

38. Thompson A, Schäfer J, Kuhn K, Kienle S, Schwarz J, Schmidt G, et al. Tandem mass tags: a novel quantification strategy for comparative analysis of complex protein mixtures by MS/MS. Anal Chem. (2003) 75:1895-904. doi: $10.1021 / \mathrm{ac} 0262560$

39. Zhu X, Gui J, Dohkan J, Cheng L, Barnes PF, Su DM. Lymphohematopoietic progenitors do not have a synchronized defect with age-related thymic involution. Aging Cell. (2007) 6:663-72. doi: 10.1111/j.1474-9726.2007.00325.x

40. Mackall CL, Punt JA, Morgan P, Farr AG, Gress RE. Thymic function in young/old chimeras: substantial thymic $\mathrm{T}$ cell regenerative capacity despite irreversible age-associated thymic involution. Eur J Immunol. (1998) 28:1886-93.

41. Gui J, Zhu X, Dohkan J, Cheng L, Barnes PF, Su DM. The aged thymus shows normal recruitment of lymphohematopoietic progenitors but has defects in thymic epithelial cells. Int Immunol. (2007) 19:1201-11. doi: 10.1093/intimm/dxm095

42. Chen L, Xiao S, Manley NR. Foxn1 is required to maintain the postnatal thymic microenvironment in a dosage-sensitive manner. Blood. (2009) 113:567-74. doi: 10.1182/blood-2008-05-156265

43. Cheng L, Guo J, Sun L, Fu J, Barnes PF, Metzger D, et al. Postnatal tissuespecific disruption of transcription factor FoxN1 triggers acute thymic atrophy. J Biol Chem. (2010) 285:5836-47. doi: 10.1074/jbc.M109.072124

44. Ortman CL, Dittmar KA, Witte PL, Le PT. Molecular characterization of the mouse involuted thymus: aberrations in expression of transcription regulators in thymocyte and epithelial compartments. Int Immunol. (2002) 14:813-22. doi: 10.1093/intimm/dxf042

45. Rode I, Boehm T. Regenerative capacity of adult cortical thymic epithelial cells. Proc Natl Acad Sci USA. (2012) 109:3463-8. doi: 10.1073/pnas.1118823109

46. Venables T, Griffith AV, DeAraujo A, Petrie HT. Dynamic changes in epithelial cell morphology control thymic organ size during atrophy and regeneration. Nat Commun. (2019) 10:4402. doi: 10.1038/s41467-019-11879-2

47. Sakata M, Ohigashi I, Takahama Y. Cellularity of thymic epithelial cells in the postnatal mouse. J Immunol. (2018) 200:1382-8. doi: 10.4049/jimmunol.1701235

48. Griffith AV, Venables T, Shi J, Farr A, van Remmen H, Szweda L, et al. Metabolic damage and premature thymus aging caused by stromal catalase deficiency. Cell Rep. (2015) 12:1071-9. doi: 10.1016/j.celrep.2015. 07.008

49. Ki S, Park D, Selden HJ, Seita J, Chung H, Kim J, et al. Global transcriptional profiling reveals distinct functions of thymic stromal subsets and agerelated changes during thymic involution. Cell Rep. (2014) 9:402-15. doi: 10.1016/j.celrep.2014.08.070

50. Wu H, Qin X, Dai H, Zhang Y. Time-course transcriptome analysis of medullary thymic epithelial cells in the early phase of thymic involution. Mol Immunol. (2018) 99:87-94. doi: 10.1016/j.molimm.2018.04.010

51. Cowan JE, Malin J, Zhao Y, Seedhom MO, Harly C, Ohigashi I, et al. Myc controls a distinct transcriptional program in fetal thymic epithelial cells that determines thymus growth. Nat Commun. (2019) 10:5498. doi: 10.1038/s41467-019-13465-y

52. Sun L, Li H, Luo H, Zhao Y. Thymic epithelial cell development and its dysfunction in human diseases. Biomed Res Int. (2014) 2014:206929. doi: 10.1155/2014/206929

53. Robles AI, Larcher F, Whalin RB, Murillas R, Richie E, Gimenez-Conti IB, et al. Expression of cyclin D1 in epithelial tissues of transgenic mice results in epidermal hyperproliferation and severe thymic hyperplasia. Proc Natl Acad Sci USA. (1996) 93:7634-8. doi: 10.1073/pnas.93.15.7634

54. Klug DB, Crouch E, Carter C, Coghlan L, Conti CJ, Richie ER. Transgenic expression of cyclin D1 in thymic epithelial precursors promotes epithelial and T cell development. J Immunol. (2000) 164:1881-8. doi: 10.4049/jimmunol.164.4.1881

55. Garfin PM, Min D, Bryson JL, Serwold T, Edris B, Blackburn CC, et al. Inactivation of the $\mathrm{RB}$ family prevents thymus involution and promotes thymic function by direct control of Foxn1 expression. J Exp Med. (2013) 210:1087-97. doi: 10.1084/jem.201 21716

56. Jenkinson WE, Bacon A, White AJ, Anderson G, Jenkinson EJ. An epithelial progenitor pool regulates thymus growth. J Immunol. (2008) 181:6101-8. doi: 10.4049/jimmunol.181.9.6101 
57. Kim M-J, Miller CM, Shadrach JL, Wagers AJ, Serwold T. Young, proliferative thymic epithelial cells engraft and function in aging thymuses. J Immunol. (2015) 194:4784-95. doi: 10.4049/jimmunol. 1403158

58. Ramond C, Berthault C, Burlen-Defranoux O, de Sousa AP, Guy-Grand D, Vieira P, et al. Two waves of distinct hematopoietic progenitor cells colonize the fetal thymus. Nat Immunol. (2014) 15:27-35. doi: 10.1038/ ni. 2782

59. Rossi SW, Jenkinson WE, Anderson G, Jenkinson EJ. Clonal analysis reveals a common progenitor for thymic cortical and medullary epithelium. Nature. (2006) 441:988-91. doi: 10.1038/nature04813

60. Bleul CC, Corbeaux T, Reuter A, Fisch P, Monting JS, Boehm T. Formation of a functional thymus initiated by a postnatal epithelial progenitor cell. Nature. (2006) 441:992-6. doi: 10.1038/nature04850

61. Ohigashi I, Zuklys S, Sakata M, Mayer CE, Zhanybekova S, Murata S, et al. Aire-expressing thymic medullary epithelial cells originate from beta5texpressing progenitor cells. Proc Natl Acad Sci USA. (2013) 110:9885-90. doi: 10.1073/pnas.1301799110

62. Rodewald HR, Paul S, Haller C, Bluethmann H, Blum C. Thymus medulla consisting of epithelial islets each derived from a single progenitor. Nature. (2001) 414:763-8. doi: 10.1038/414763a

63. Kernfeld EM, Genga RMJ, Neherin K, Magaletta ME, Xu P, Maehr R. A single-cell transcriptomic atlas of thymus organogenesis resolves cell types and developmental maturation. Immunity. (2018) 48:1258-70.e1256. doi: 10.1016/j.immuni.2018.04.015

64. Bornstein C, Nevo S, Giladi A, Kadouri N, Pouzolles M, Gerbe F, et al. Single-cell mapping of the thymic stroma identifies IL-25-producing tuft epithelial cells. Nature. (2018) 559:622-6. doi: 10.1038/s41586-0180346-1

65. Miller CN, Proekt I, von Moltke J, Wells KL, Rajpurkar AR, Wang H, et al. Thymic tuft cells promote an IL-4-enriched medulla and shape thymocyte development. Nature. (2018) 559:627-31. doi: 10.1038/s41586-0180345-2

66. Hauri-Hohl M, Zuklys S, Hollander GA, Ziegler SF. A regulatory role for TGF-beta signaling in the establishment and function of the thymic medulla. Nat Immunol. (2014) 15:554-61. doi: 10.1038/ni.2869

67. Ohigashi I, Tanaka Y, Kondo K, Fujimori S, Kondo H, Palin AC, et al. Trans-omics impact of thymoproteasome in cortical thymic epithelial cells. Cell Rep. (2019) 29:2901-16.e2906. doi: 10.1016/j.celrep.2019. 10.079

68. Nurse P. Universal control mechanism regulating onset of M-phase. Nature. (1990) 344:503-8. doi: 10.1038/344503a0

69. Lim S, Kaldis P. Cdks, cyclins, and CKIs: roles beyond cell cycle regulation. Development. (2013) 140:3079-93. doi: 10.1242/dev.091744

70. Malumbres M, Barbacid M. Mammalian cyclin-dependent kinases. Trens Biochem Sci. (2005) 30:630-41. doi: 10.1016/j.tibs.2005. 09.005

71. Hydbring $\mathrm{P}$, Malumbres M, Sicinski P. Non-canonical functions of cell cycle cyclins and cyclin-dependent kinases. Nat Rev Mol Cell Biol. (2016) 17:280-92. doi: 10.1038/nrm.2016.27

72. Quandt E, Ribeiro MPC, Clotet J. Atypical cyclins: the extended family portrait. Cell Mol Life Sci. (2019) 77:231-42. doi: 10.1007/s00018-019-03262-7

73. Sherr CG. Mammalian G1 cyclins. Cell. (1993) 73:1059-65. doi: 10.1016/0092-8674(93)90636-5

74. Ohtsubo M, Theodoras AM, Schumacher J, Roberts JM, Pagano M. Human cyclin E, a nuclear protein essential for the G1-to-S phase transition. Mol Cell Biol. (1995) 15:2612-24. doi: 10.1128/mcb.15.5.2612

75. Pagano M, Pepperkok R, Verde F, Ansorge W, Draetta G. Cyclin A is required at two points in the human cell cycle. EMBO J. (1992) 11:961-71.

76. Huang, Y, Sramkoski RM, Jacobberger JW. The kinetics of G2 and M transitions regulated by B cyclins. PLoS ONE. (2013) 8:e80861. doi: 10.1371/journal.pone.0080861

77. Quelle DE, Ashmun RA, Shurtleff SA, Kato JY, Bar-Sagi D, Roussel MF, et al. Overexpression of mouse D-type cyclins accelerates G1 phase in rodent fibroblasts. Genes Dev. (1993) 7:1559-71. doi: 10.1101/gad. 7.8.1559
78. Wang TC, Cardiff RD, Zukerberg L, Lees E, Arnold A, Schmidt EV. Mammary hyperplasia and carcinoma in MMTV-cyclin D1 transgenic mice. Nature. (1994) 369:669-71. doi: 10.1038/369669a0

79. Deane NG, Parker MA, Aramandla R, Diehl L, Lee WJ, Washington $\mathrm{MK}$, et al. Hepatocellular carcinoma results from chronic cyclin D1 overexpression in transgenic mice. Cancer Res. (2001) 61:5389-95.

80. Gansauge S, Gansauge F, Ramadani M, Stobbe H, Rau B, Harada N, et al. Overexpression of cyclin D1 in human pancreatic carcinoma is associated with poor prognosis. Cancer Res. (1997) 57:1634-7.

81. Shan YS, Hsu HP, Lai MD, Hung YH, Wang CY, Yen MC, et al. Cyclin D1 overexpression correlates with poor tumor differentiation and prognosis in gastric cancer. Oncol Lett. (2017) 14:4517-26. doi: 10.3892/ol. 2017.6736

82. Robles AI, Conti CJ. Early overexpression of cyclin D1 protein in mouse skin carcinogenesis. Carcinogenesis. (1995) 16:781-6.

83. Klug DB, Carter C, Crouch E, Roop D, Conti CJ, Richie ER. Interdependence of cortical thymic epithelial cell differentiation and T-lineage commitment. Proc Natl Acad Sci USA. (1998) 95:11822-7. doi: 10.1073/pnas.95.20. 11822

84. Bolner ML. Preventing thymus involution in K5.Cyclin D1 transgenic mice sustains the naive $T$ cell compartment with age (UT GSBS Dissertations and Theses), The University of Texas, Austin, TX, United States (2015). p. 636.

85. Matloubian M, Lo CG, Cinamon G, Lesneski MJ, Xu Y, Brinkmann $\mathrm{V}$, et al. Lymphocyte egress from thymus and peripheral lymphoid organs is dependent on S1P receptor 1. Nature. (2004) 427:355-60. doi: $10.1038 /$ nature 02284

86. Surh CD, Sprent J. Homeostasis of naive and memory T cells. Immunity. (2008) 29:848-62. doi: 10.1016/j.immuni.2008.11.002

87. Murata S, Sasaki K, Kishimoto T, Niwa S, Hayashi H, Takahama Y, et al. Regulation of CD8 $+\mathrm{T}$ cell development by thymus-specific proteasomes. Science. (2007) 316:1349-53. doi: 10.1126/science.1141915

88. Nitta T, Murata S, Sasaki K, Fujii H, Ripen AM, Ishimaru N, et al. Thymoproteasome shapes immunocompetent repertoire of CD8+ $\mathrm{T}$ cells. Immunity. (2010) 32:29-40. doi: 10.1016/j.immuni.2009. 10.009

89. Takada K, Van Laethem F, Xing Y, Akane K, Suzuki H, Murata S, et al. TCR affinity for thymoproteasome-dependent positively selecting peptides conditions antigen responsiveness in CD8+ T cells. Nat Immunol. (2015) 16:1069-76. doi: 10.1038/ni.3237

90. Mathis D, Benoist C. Aire. Annu Rev Immunol. (2009) 27:287-312. doi: 10.1146/annurev.immunol.25.022106.141532

91. Nakagawa Y, Ohigashi I, Nitta T, Sakata M, Tanaka K, Murata S, et al. Thymic nurse cells provide microenvironment for secondary TCR $\alpha$ rearrangement in cortical thymocytes. Proc Natl Acad Sci USA. (2012) 109:20572-7. doi: 10.1073/pnas. 1213069109

92. Thompson HL, Smithey MJ, Uhrlaub JL, Jeftic I, Jergovic M, White $\mathrm{SE}$, et al. Lymph nodes as barriers to T-cell rejuvenation in aging mice and nonhuman primates. Aging Cell. (2019) 18:e12865. doi: 10.1111/acel. 12865

93. Aronson M. Hypothesis: involution of the thymus with aging-programmed and beneficial. Thymus. (1991) 18:7-13.

94. Xia J, Wang H, Guo J, Zhang Z, Coder B, Su DM. Age-related disruption of steady-state thymic medulla provokes autoimmune phenotype via perturbing negative selection. Aging Dis. (2012) 3:248-59.

95. Coder BD, Wang H, Ruan L, Su DM. Thymic involution perturbs negative selection leading to autoreactive $\mathrm{T}$ cells that induce chronic inflammation. J Immunol. (2015) 194:5825-37. doi: 10.4049/jimmunol.15 00082

96. Quinn KM, Zaloumis SG, Cukalac T, Kan WT, Sng XY, Mirams M, et al. Heightened self-reactivity associated with selective survival, but not expansion, of naïve virus-specific CD8+ $\mathrm{T}$ cells in aged mice. Proc Natl Acad Sci USA. (2016) 113:1333-8. doi: 10.1073/pnas.15251 67113

97. Goronzy JJ, Weyand CM. Immune aging and autoimmunity. Cell Mol Life Sci. (2012) 69:1615-23. doi: 10.1007/s00018-012-0970-0

98. Klein L, Kyewski B, Allen PM, Hogquist KA. Positive and negative selection of the T cell repertoire: what thymocytes see (and don't see). Nat Rev Immunol. (2014) 14:377-91. doi: 10.1038/nri3667 
99. Dowling MR, Hodgkin PD. Modelling naive T-cell homeostasis: consequences of heritable cellular lifespan during ageing. Immunol Cell Biol. (2009) 87:445-56. doi: 10.1038/icb. 2009.11

100. Dowling MR, Hodgkin PD. Why does the thymus involute? A selection-based hypothesis. Trends Immunol. (2009) 30:295-300. doi: 10.1016/j.it.2009.04.006

101. Hakim FT, Memon SA, Cepeda R, Jones EC, Chow CK, Kasten-Sportes $\mathrm{C}$, et al. Age-dependent incidence, time course, and consequences of thymic renewal in adults. J Clin Invest. (2005) 115:930-9. doi: 10.1172/JCI 22492
Conflict of Interest: The authors declare that the research was conducted in the absence of any commercial or financial relationships that could be construed as a potential conflict of interest.

Copyright (c) 2020 Cowan, Takahama, Bhandoola and Ohigashi. This is an openaccess article distributed under the terms of the Creative Commons Attribution License (CC BY). The use, distribution or reproduction in other forums is permitted, provided the original author(s) and the copyright owner(s) are credited and that the original publication in this journal is cited, in accordance with accepted academic practice. No use, distribution or reproduction is permitted which does not comply with these terms. 